\title{
Aspect Oriented Middleware for Internet of Things: A State-of-the Art Survey of Service Discovery Approaches
}

\author{
Senthil Murugan Balakrishnan ${ }^{1}$, Arun Kumar $\operatorname{Sangaiah}^{2 *}$ \\ ${ }^{1}$ School of Information Technology and Engineering, VIT University, Vellore, Tamil Nadu, India \\ ${ }^{2}$ School of Computing Science and Engineering, VIT University, Vellore, Tamil Nadu, India \\ *Email: senthilmurugan.b@vit.ac.in ${ }^{1}$, sarunkumar@vit.ac.in ${ }^{2}$
}

\begin{abstract}
Internet of Things refers to a paradigm consisting of a variety of uniquely identifiable day to day things communicating with one another to form a large scale dynamic network. The integration of these heterogeneous things in the constrained network is a challenging issue, for which service oriented and architecture based solutions are considered to be useful. The key element of any service oriented paradigm is service discovery which allows the client to get access to the right service at the right time to complete the requested tasks. This paper presented a comprehensive survey of major solutions for service and resource discovery detailing techniques and features used in existing systems. The survey presented the findings in tabular representation and outlined the proposed aspect oriented middleware framework for IoT highlighting context awareness and solving certain pitfalls in the existing service discovery approaches. The survey results might be helpful in deriving the limitations of the existing protocols in literature and identify future research opportunities in service discovery.
\end{abstract}

Keywords: Internet of Things (IoT); Service discovery; Resource discovery; CoAP; P2P; Jini; Bluetooth; SDP.

\section{Introduction}

The role of Internet of Things (IoT) is paramount in the shift of Internet to Future Internet. Over the years Internet has become the most vital and popular network that enables everyone to create, generate, share, use and combine the information from every corner of the world and produce knowledge from it. Now Internet has been evolved into Future Internet in which the services are federated and autonomic networks that provide built-in support for service management, contextualization, mobility, security and resilience are embedded [1]. Future Internet can be defined as the union and cooperation [2] of Internet of Content to promote the creation and consumption of media content of any type and volume [4], the Internet of Services to demonstrate the scenarios of services creation and the operation in the internet [5] and Internet of Things (IoT) to link the physical and virtual objects through communication capabilities
[3]. The "Internet of Things" paradigm aims at providing models and mechanisms enabling the creation of networks of "smart things" on a large scale by means of RFID, wireless sensor and actuator networks, where embedded devices are distributed in the physical environment [6]. IoT represents a system in which the various technologies like ubiquitous computing, pervasive computing, internet protocol, sensor networks, embedded systems and communication technologies are merged together and where the real and digital world will meet and interact. The smart objects are considered to be the basic building block of IoT. By putting intelligence into the day to day things we see they are turning into smart objects and through internet they are able to interact, coordinate, exchange data and information with the physical world [7].

IoT is a global network infrastructure linking physical and virtual objects through the exploitation of data capture and communication capabilities. The infrastructure includes existing and evolving Internet 
and network developments. It will offer specific object-identification, sensor and connection capability as the basis for the development of independent cooperative services and applications. These will be characterized by a high degree of autonomous data capture, event transfer, network connectivity and interoperability [8]. IoT aims at connecting billions of smart devices to form an internet like structure and facilitating interactions among things and people. Smart objects are the type of constrained devices with the constraints on their memory size, computing power, communication capabilities and or available power [9]. This smart object is equipped with microcontrollers, a radio interface, sensors and or actuators and offers the functionality as services. The abstraction of resources as services has opened IoT to the excess of potential applications [10]. The adopted web service paradigm is useful for promoting interoperability among the heterogeneous devices by offering a standard interface to interact with others. Discovering these services in the network is crucial when providing application level end to end integration. The broad categories of approaches for service discovery exists in the literature, in which traditional methods are not suitable for smart environment due to the fact that it requires heavy communication and large data format and registry based approach incurs excess traffic in maintaining the availability status of the device. The research need in the aspect of service discovery is the development of feasible solution enabling wide application coverage of these networks. Realization of IoT requires the conception and development of dynamic adaptation and autonomic capabilities. This initiates the need for developing the mechanisms for discovering available resources and capabilities.

Considering the importance of service discovery in these emerging trends, we have developed the understanding of various service discovery protocols currently in existence. This paper is organized as follows: in Section 2 of this paper, a brief review of service discovery technique and various approaches involved therein are discussed. Section 3 of this paper presents the comprehensive survey of leading technologies in service discovery methods highlighting the functional features and implementation issues. The findings in the survey are provided in Section 4. Section 5 describes the proposed middleware framework for addressing issues with service discovery approaches and finally Section 6 concludes the paper.

\section{Service Discovery}

Service discovery is the process of identifying suitable devices according to the service description submitted by the requestor. The identification process [11] involves locating the requested services, retrieving the service description of those services and executing the matchmaking algorithm between those descriptions and request and finally obtaining the description that perfectly matches the request. The service descriptions are description language that is specific to protocols to define keywords and syntax used to describe services and its properties. The description format [12] may be either key/value, template based or semantic description based.

The requirements involved in the design of service discovery protocol from the service provider and consumer perspective can be broadly categorized as functional and non-functional [13]. The data structures and methods involved therein are main constituents of the functional requirements for service provider. These methods are for publishing, updating and un-publishing the discovery service description. Service description consists of name value pairs describing the attributes and there is no limit imposed over the number of attributes. The functional requirement from the consumer side is the provision of suitable methods to enable querying the discovery service. The end users should be able to specify the upper bound on the number of services to be returned and provide a means for iterating them. The non-functional requirements include the attributes such as low response time, reliability, scalability and so on.

The service discovery protocols are generally classified as centralised, distributed and hierarchical approaches. Centralised approach relies on a directory, in which Service Agents (SA) registers their services and User Agents (UA) discovers the services by sending the unicast queries to the directory. In distributed approach the participant nodes communicate through multicasting the queries for discovering the resources. Hierarchical method includes the cluster of nodes with high capabilities.

Service discovery architectures are of two types namely directory based and directory less [19]. In the directory based model, there will be a dedicated logically centralized and physically distributed directory to maintain the whole service description. These directories are responsible for registering service descriptions and handling user queries. In the latter the individual service provider maintains the service description and is responsible for processing and replying to the incoming queries. 


\subsection{Service discovery issues}

Issarny et al. [2] discusses the service discovery from the viewpoint of issues and concerns related to the organization of service description in registries and protocols used for service retrieval. In the context of information organization, the baseline is the proposal of data model in Universal Description, Discovery and Integration (UDDI) specification [42]. According to UDDI, business entities announce their offerings in white pages, taxonomy based advertisements appear in yellow pages and pointers to web service descriptions are provided in green pages. UDDI proposes the usage of tModels to provide the technical information about web services. The main research challenge lying in the service discovery field is the augment of the existing simple UDDI with the semantically rich metadata and the sophisticated querying techniques. Another research challenge in the management of service description is in dealing with heterogeneity of service descriptions.

As far as the service retrieval protocols are concerned, the baseline approach is centralized in which the single registry maintains the information about service description thereby facilitating fast access. The pitfall in the centralized approach is that it does not scale well with respect to the number of users submitting the queries and prone to single point of failure. To overcome these difficulties, decentralized solutions called pure distribution and hybrid approaches are proposed. In the purely distributed method, participating nodes in the network stores store the registry locally and distributed querying service spans these registries to compile and present the required information to the user. The drawback of this method is the communication overhead involved in establishing a contact between peers and same is surmounted by adopting multicasting solution instead of broadcasting, [52]. Considering the scalability issues in multicasting domain [43], the solution in terms of minimizing the number of search hops is proposed [44] and the performance selective forwarding $[44,45,46]$ in which the peers that are not able to solve the service discovery query uses the previous knowledge to forward the query to the correct peers. An alternative to selective forwarding is sending the queries to the peer for which the probability decreases with the number of prior queries that remain unanswered [47]. The hybrid approach contains a super peer that acts yellow pages within the $\mathrm{P} 2 \mathrm{P}$ network. It is mostly used in the mobile environment and the effectiveness of using it in resolving the service discovery queries is achieved by reduction in messaging overhead and better response time.

Another important issue related to the service discovery protocols in the heterogeneity. The research ideas evolved for solving the heterogeneity is to employ the middleware layer on the top of the heterogeneous protocol responsible for mapping the service discovery queries that conform to a particular protocol to service discovery queries that conform to other protocols. Some of the examples are ReMMoC[48], INDISS [49], MUSDAC [50].

Finally security, privacy and reliability are also important concerns in the context of service discovery. The study in [51] lists the various requirements that should be satisfied by the service discovery protocols for achieving security. Comparison of well-known service discovery protocols like Jini, SLP etc clearly state that these protocols partially satisfied the aforementioned requirements [51].

\subsection{Research method}

The fundamental step towards the research was the identification of research questions through extensive survey. This study was undertaken as a systematic literature review to formulate the research problem in the Internet of Things paradigm. Some basic questions addressed are as follows

Q1: Whether service discovery/resource discovery is assumed as a challenging problem by the academicians/practitioners?

Motivation: The purpose is to get an idea about the importance of service/resource discovery in Internet of Things field.

Q2: What are the available methods for service/resource discovery?

Motivation: To develop a broad knowledge of the existing methods in order to be able to refine these methods or propose a new method.

Q3: What are the commonalities, comparisons between the approaches?

Motivation: To develop a comparative view of various methods and to get an idea of how these approaches cooperate and interact with each other.

\section{Literature from Past Research Works}

Many service discovery approaches are available in the literature, to name a few UPnP, Bluetooth SDP, SLP, Bonjour and Salutation. Universal Plug and Play (UPnp) [23] protocol is used in small office and home to create a device to device network. It uses TCP as transport protocol and XML as service 
description format. The main components of UPnP are control points or service directories and devices. The service discovery in UPnP works according to Simple Service Discovery Protocol (SSDS) [24] that operates using HTTP over multicast and unicast UDP. The drawback of using UPnP is that it does not scale well for the multicasting scenario and lacks support for attribute based querying for services.

Jini [25] is a service discovery architecture for demonstrating the interaction between Java enabled devices. The central component of Jini is lookup servers that are capable of storing the service descriptions published by the service providers and answering the client queries. The publishing parameters include the service objects along with their attributes. This service object is proxies written in Java and exposes a remote interface that is accesible by client. The client request contains the specification about type of service requested and its associated attributes. Jini is augmented with various features like leases by which those services not updated for a longer time are deleted from a registry.

Another service discovery framework for home and enterprise called Salutation [27] allows devices and services in the network to discover each other and advertise their capabilities. The primary component of Salutation is Salutation Manager (SLM) which is present in each participating device in the network. SLM uses Salutation Manager Protocol to discover services available in other devices.

The service discovery architecture for the Bluetooth enabled devices is Bluetooth SDP[28]. The characteristics of the services are represented as service record consisting of attribute-value pairs. Bluetooth SDP follows service searching and service browsing methods for service discovery. With the service searching method, the requestor generates the query containing service attributes and in turn gets the records that match the one from the provider side. With the second method, the client passes the generic query and gets a list of all services of a specific provider. The major drawback is its limited discovery capability due to 1-hop discovery support.

Another mechanism named Service Location Protocol (SLP) [34] is an IETF standard used in large scale IoT scenarios through which computers and devices can find services in LANs without prior configuration. Services in the network consist of attribute value pairs and are uniquely identified using URLs. The client's queries for service finding is processed using the substring matching feature of SLP. It supports service browsing and multicasting communication by which the Directory Agents (DA) multicast a signal to all the available Service Agents (SA) on the provider's side. SA's hearing the signal registers itself with DAs. User Agents (UA) at the client side unicast their queries to the DA for the service description retrieval. In the absence of DA, UAs multicasts their queries and all the receiving SAs whose service description matches with the queries respond in unicast mode. The drawback in this model is that SLP follows centralized approach due to that they are failure prone and are not used in constrained devices.

The service discovery protocol adopted by Bonjour [37], an Apple technology to discover services and devices in the network, is based on Multicast DNS Service Discovery by which hosts in an adhoc network resolve host names and service names to IP addresses without relying on the DNS servers. In Bonjour client multicasts the queries specifying the service type, domain and the communication protocol desired, for which the service providers respond by DNS service records. The new providers have entered into the network that multicasts their presence to all devices to create awareness.

The earlier study [21] proposes a mechanism named as "discovery" for the global discovery of devices and sensors. The methodology offers the framework that allows user to register their own devices into the infrastructure and discovers the available resources through mobile phone. The primary focus of this research work is on the discoverability of devices based on context aware- ness and geolocation. The framework developed is scalable and supports a variety of devices, domains and integration of heterogeneous technologies that include RFID, sensor networks, Bluetooth, and WiFi. The architecture is further augmented with the application to discover services anywhere in the world and search engine support to perform operations like query, filtering and search. The novelty in the work is firstly the application discovering sensors and services in the surrounding geolocation and context awareness capabilities through the deployed backend or through RFID/QR codes; secondly the capabilities to integrate and support legacy technologies, thirdly the integration of EPCIS (Electronic Product Code Information System) to identify physical objects as a smart thing, and finally the ability to gather geolocation information thereby supporting high context awareness. The framework relies on the centralized solution exposing a search engine to make the integration of distributed services directories feasible.

The existing internet architectures in the literature are based on host to host connectivity suitable for the sharing of resources rather than data. These architectures are not to be applied in the IoT field, as 
the major concern of IoT is in sharing of data. Peer-to-Peer systems [14] represent a content centric internet technology fit for IoT and are useful for implementing an effective mechanism for discoring available resources and capabilities in the network. The structured approaches based on Distributed Hash Tables (DHT) are scalable, resilient and efficient in terms of distributing the load among the peers. The solutions like Prefix Hash Tree (PHT) [15], Mercury [16], MAAN [17] and Squid [18] are providing significant contributions in handling complex queries in IoT service discovery.

The resource discovery mechanism in [22] is a peer-to-peer discovery model that is different from conventional peer-to-peer resource discovery approaches for IoT in which only single attribute matching is supported. The proposed methodology adopts DHT indexing scheme and guarantees scalability, robustness and maintainability of the system and supports multi-attribute and range queries. The layered design consists of an SFC linearization technique for mapping a multidinsional keyword space into a one dimensional index space, Prefix Hash Tree (PHT) search structure for implementing DHT get/put interface and DHT implementation based on Kademlia algorithm. This model exploits the distributed data management capabilities offered by this peer-to-peer overlay network to build application specific APIs for search and management of resource discovery information. Computational simulation estimates average leaf depth of the trie and the average block utilization for the dangerous goods monitoring application in the industrial monitoring domain. In addition, a previous researcher [22] claims that the proposed layered solution privileges ease of design and implementation compared to other overlay dependent indexing schemes. The focus of the developed model is in the aspect of service resolution rather than on the creation of overlay by automatically discovering existing services.

Constrained Application Protocol (CoAP) [40], a main work from CoRE Working Groups supports built-in discovery of services and resources which are extremely important for machine-to-machine applications to avoid human intervention. The manual configuration is required only at the point of creating a CoRE link format for discoverable resources. Each CoAP server in the participating network exposes an interface called /.well-known/core to which the other nodes in the network send requests for discovering available resources. The return message from the server consists of lists of resources and the content-format code "ct" for each resource that provides the hint about the format of the data associated with the resource. In CoAP, the newly joining node in the network is not informed about how it should announce itself to the requestor nodes.

The specification in [26] dictates the implementation of CoAP on top of the IP multicast to derive the usage of CoAP in group communication context. Multicast resource discovery is required to locate the resources residing in the scope that supports IP multicast. The request to multicast IP address is generated using HTTP GET and directed to /.well-known/core interface. The proposed mechanism works only within an IP multicast domain and does not scale to larger networks that do not support end-to-end multicast.

The centralized approaches for service and resource discovery are algorithmically simple but suffer from severe drawbacks in the aspect of scalability and single point of failure. CoAP follows centralized approach for service and resource discovery, due to the fact that it lacks scalability and availability and is prone to denial-of-service (DOS) attacks. To overcome these limitations and to scale to the large extent, DHT based approaches are used as alternatives in the literature.

$\mathrm{Ni}$ Yulinet. al [29] propose $\mathrm{P} 2 \mathrm{P}$ based distributed UDDI web service discovery methodology to overcome the single point of failure and performance bottleneck problems faced in the UDDI based centralized approach. The proposed model uses Chord, a highly scalable and highly efficient structured P2P protocol to organize distributed UDDI. Distributed UDDIs provides necessary functional modules for publishing web services by providers, looking up of the required services by service requestors, storing the service index, routing the service index to appropriate UDDI and communicating with other distributed UDDIs.

Sven Kaffille et al [30] propose a protocol for decentralized service discovery with the guarantee of finding any service description to match a given query without having to flood the whole network which is inherently inefficient. The approach uses peer-to-peer overlay network based on Chord DHT. The proposed P2P discovery service consists of three layers namely transport layer - the lowest layer, P2P overlay network Chord and local discovery service layer. On the top of the local discovery service layers the application may be built. Authors compared the simulation of the proposed protocol with the Gnutella like network with various assumptions in the features such as network size and dynamics. However, these approaches do not take 
into account the constraints and requirements for IoT.

Peter Wright et al. [31] propose a two phase constraints based resource discovery model which operates in a multi-provider cloud environment. In the first phase a set of infrastructure resources are identified and in the second phase a heuristic is applied to select the most appropriate resources from the set. The model classifies application's constraints according to user's need into hard and soft constraints. Hard constraints are 'must-have' requirements which persist and are not changeable during execution, whereas soft constraints are requirements thatcan change. Utilizing only the hard constraints, the set of possible resources are generated during the initial phase. Secondly it applies suitable heuristic that can give weights to the various contributing factors to obtain an application centric metric. Once the metric is identified, the model selects the optimal solution path that matches the application requirements.

Simone Ciraniet. al [36] develop a automated service and resource discovery mechanism for large scale IoT networks. It uses $\mathrm{P} 2 \mathrm{P}$ technology to provide a distributed large scale service discovery and Zeroconf methodology to realize the discovery at the local scale. The proposed approach is scalable, fault tolerant and self-configuring in nature due to the adoption of $\mathrm{P} 2 \mathrm{P}$ architecture and the absence of content related dependencies that makes it robust and avoids application specific constraints. This methodology makes use of special node called "IoT gateway", - a boundary node to store information on the resources provided by smart devices in the local wireless network. IoT gateway acts as a HTTP-to-CoAP proxy by translating HTTP requests to CoAP requests and vice versa. Service discovery module gets the port number of the CoAP servers in the network and the resource discovery discovers the resources that the CoAP server manages. Whenever IoT gateway detects the arrival of new CoAP node it asks for the list of supported services. The stored information is then used to route the incoming requests to the appropriate resource node. These IoT gateways are federated in a $\mathrm{P} 2 \mathrm{P}$ overlay to perform large scale resource discovery.

Service discovery in [38] uses propagation like query/response model, minimalist access control policy and is based on the friends of my friends are my friends principle. It uses blacklist/whitelist strategy in which the blacklist contains the service providers with whom the communication is not to be done and whitelist contains the required collaborators. The service requestor broadcasts the service description only to the providers in the whitelist and in turn the receiver responds with the services whose description matches with the request. Subsequently, these receivers forward the request to the list of providers maintained in its whitelist and it is recursively propagated. Finally, the requestor will receive more service responses and proceeds with the service selection phase. The approach mainly concentrates on enforcing security in terms of access control.

Recent research works on promoting interoperability in IoT networks proposes the use of semantic web technologies for discovering the sensor services and data in IoT environment [53] [54]. Enabling business based Internet of Things (ebbits) [32], an European Commission project aiming to develop architecture, technology and processes allows businesses to semantically integrate the Internet of Things into mainstream enterprise systems and supports interoperable real-world, online end-to-end business applications. The resource discovery features available in ebbits include semantic discovery for local entity resolution and attribute based discovery for global resolution. Semantic discovery relies on OWL Lite based ontology to model the device services, device capabilities, quality of service aspects and applications. The global service discovery leverages on attribute based description of services and takes privacy into account so that the query containing attribute names and values is broadcasted into the network and the intended person holding the searched attributes understands the message and answers it and for others who are computationally hard to decide about the searched attributes.

OpenIoT [41] embeds the cloud computing feature into IoT so that IoT services can be delivered on demand, as cloud seems to be the natural home for IoT applications [20]. It offers a middleware solution for the creation of sensor cloud infrastructure. OpenIoT facilitates the storage of sensor and Internet Connected Objects (ICO) data into the cloud and provides strategies for on-demand selection of sensors and data streams. The sensors and ICOs in OpenIoT are connected with the cloud system with the help of Global Sensors Networks (GSN) open source middleware and are assigned with unique URI (Universal Resource Identifier). OpenIoT uses semantic web technologies to implement applications that span across internet and embed technology domain [35]. OpenIoT uses semantically annotated ICO that conforms to OpenIoT ontology based on W3C SSN (Semantic Sensors Network) and SPARQL language for performing resource discovery. OpenIoT relies on the registration of sensors with the directory services 
for discovery purpose. Discovery is performed by searching the directory services for the list of triples that match against the SPARQL query.

The resource constrained, discontinuous disconnections and mobility nature of devices enables the services in IoT as highly dynamic and leads to formulate different approaches in the aspect of context awareness compared to traditional context aware web service discovery methods. Qiang Wei et al [33] present a context aware resource discovery architecture for IoT adaptable to highly dynamic environment considering temporal dimensions and provide environment aware services to things with minimal human intervention. The proposed service discovery mechanism analyzes the role of context and relations with entities in the IoT environment, based on which a common ontology representation addressing uncertainties and temporal aspects of the context is developed. Dynamic Bayesian Network (DBN) is used as reasoning approach to reason about the context to sense current situation and provides support for enabling smart services to things. The findings are most suitable for the environments with limited scopes and not for pervasive computing field.

Talal Ashraf Butt [39] proposes a RESTful web services based service discovery solution called TRENDY to overcome the problems introduced in the context of interoperability in constrained domains. The proposed method uses demand based adaptive timer and caching mechanism to reduce the communication overhead and service invocation delay. It is a registry based service discovery solution with context information thereby it uses the context information to provide optimal service selection.

\section{Research Findings from the Review of Li- terature}

In this research, we have summarized the observations with respect to the research gaps from literature review as depicted in Table 1. Further, Table 1 shows the summary of some recent approaches for service discovery highlighting the purpose and features embedded in it.

From the research perspective, the analysis of the service discovery protocols discussed above exposedthe challenges in the aspect of services availability, heterogeneity, scalability, and topological information and so on. The research view droped down to explore the possibilities of developing the smart solution in terms of middleware to overcome the hurdles in the design and imple- mentation of these protocols. The analysis further revealed that the IoT systems are likely to be context dependent and pervasive in nature, hence the requirements that services have to satisfy change continuously. Therefore, the middleware development methodology is based on Aspect oriented software development in which the functionalities are modelled as aspects and are activated as need arises and facilitates reconfiguration.

\section{Our Proposed Middleware Framework for IoT}

The aforementioned studies of various service discovery protocols in the literature discuss the issues and concerns in the aspect of implementation and usage of these protocols for smart environment. In general, IoT derives various challenges from the Internet in the context of scalability, heterogeneity, undefined topology and data point information, incomplete metadata and conflicts in user preferences. The non-availability of data point information is one of the consequences of unknown topology details in which no more suitable device is available at the geographical site to provide required information or the device has not collected or stored the data point required. There is a strong need to estimate these missing data points at a greater degree of accuracy without human intervention. Similarly, the information related to the services has been manually entered by the human operator at the installation time. As the devices strength grows larger, the manual entries result in inaccurate and incomplete information. This necessitates the need for methodology to recover these missing details at the required time.

To address the issues involved in undefined data point and inaccurate metadata through the discovery of service information, we investigated on developing a middleware for an IoT based system. The middleware acts as an interface between the IoT hardware and the application to process the data gathered from the device component and is responsible for interacting with IoT devices, managing the information associated [56] and solving heterogeneity issues [57]. The proposed middleware is incorporated with the service that performs functional substitution thereby avoiding manual intervention in certain situation, for example in the event of computing the wind-chill factor at a certain location where the wind-chill sensors or actuators are not available for providing data about the needed location. Then the system is expected to compute the desired information through the composition of the 
temperature and wind speed readings using conventional wind chill equation, as the literature reveals that the function of a wind-chill sensor is equivalent to the function provided by the thermometer [58]. The middleware is augmented with the services that uses the required estimations in the event of non-availability of data and distributes monitoring feature to avoid possible missing of data. For instance, if an application would like to know about the temperature at a particular location where no thermometer exists, then the middleware service should estimate the result based of the values of the temperature readings of neighborhood location.

The smart environment is equipped with devices that vary with respect to memory, processing capabilities and connectivity. This requires that the middleware component itself should be reconfigured dynamically in the aspect of runtime deployment and reconfiguration of services thereby making it possible to add, remove, and replace the services during running. Moreover the services are distributed in nature, which enables the non functional requirements like security and scalability to be taken into account for implementation. The proposed middleware is designed to incorporate the dynamic reconfiguration capability in order to properly adapt to the continuous changes in the environment and situation aware services to provide user support.

The research output of this survey article is to emphasize the creation of single proposal that solves the issues concerned with all the requirements listed aboveby adopting the novel approach for the development of systems thereby achieving effecttiveness in the design. Aspect Oriented Software Development (AOSD) technology is found to be most appropriate for these needs. In AOSD the functionalities are considered as aspects and are dynamically enabled or disabled based on the situation [55]. Furthermore, the services rendering the functionality can also be replaced by alternative implementation that suits the current context. The middleware proposed is conceived as web of agents to overcome the challenge involved in generating inaccurate data and missing data. Lastly the middleware and the agents embodied in it help the distributed software system for IoT to be able to adapt to meet changing user needs and to manage the variability of the open environment they operate in. Finally, the research goal is to design and implement a self-adaptive aspect oriented middleware that performs context monitoring and dynamic reconfiguration to adapt to changes in the currently executing IoT environment addressing all the requirements mentioned above.

Thiago et al. [58] suggest five layered archi- tecture for the IoT. Those layers include Edge Technology layer which consists of RFID tags, sensors, actuators and hardware devices constituting things in the IoT paradigm, Access Gateway Layer dealing with publishing, subscribing services provided by the things, message routing and communication, Internet layer for connecting source and destination, Middleware layer including functionalities like services discovery, service selection and security, and application layer to deliver the services. Our envisioned aspect oriented IoT middleware is depicted in Figure 1. The contributions of our proposed work are threefold as follows:

- Design and implementation of aspect oriented middleware

- Multi-agents to introduce the functionalityof monitoring the running environment and adapts in response to changing user and environmental changes by running new configuration

- Deploying functional substitution and on-demand estimation services as aspects in middleware and enablingdisabling whenever necessary.

The critical parts of the proposed middleware framework include smart environment monitoring service (SeMS), reconfiguration service for adaptability(RSA) and aspect management service (AMS). RSA contains the required logic for reconfiguration based on the context data supplied by the SeMS. SeMS consists of mobile agents and intelligent agents for smart environment monitoring and extracting the information. Agents are capable of moving and distributing themselves to perform their monitoring task lead. They are used to solve problems by using a decentralized approach where several agents contribute to the solution by cooperating each other. The primary advantage of software agents is the intelligence that can be embodied into them according to some collective artificial intelligence approach that needs cooperation among several agents that can run on a parallel or distributed computer to achieve the needed high performance for solving large complex problems keeping execution time low. AMS helps in woven and unwoven of functionality modelled as aspects. Once we have developed the main services of the middleware, sample application scenarios suitable for IoT environment will be designed and evaluated. The application services are reconfigured based on the context information provided by the monitoring agents and appropriate functional components are substituted based on the context data. Based on the context data and service description 
the configuration repository is populated. The RSA

on the context data.

module will select appropriate configuration based

Table 1 Comparison of Service Discovery Approaches

\begin{tabular}{|c|c|c|c|c|c|}
\hline \multirow{2}{*}{ Year } & \multirow{2}{*}{ Publication Title } & \multicolumn{3}{|c|}{ Methods for Service Discovery } & \multirow{2}{*}{$\begin{array}{c}\text { Centralized/ } \\
\text { Distributed }\end{array}$} \\
\hline & & 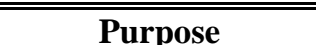 & Approach & $\begin{array}{l}\text { Research Gaps } \\
\end{array}$ & \\
\hline 2014 & $\begin{array}{c}\text { Mobile digcovery: } \\
\text { discovering and } \\
\text { interacting with the } \\
\text { world through the } \\
\text { Internet of things. [21]. }\end{array}$ & $\begin{array}{l}\text { Global Discovery of } \\
\text { devices and sensors }\end{array}$ & $\begin{array}{l}\text { Discovery based on } \\
\text { context awareness and } \\
\text { geo-location }\end{array}$ & $\begin{array}{l}\text { Handling } \\
\text { Uncertainties }\end{array}$ & Centralized \\
\hline 2014 & $\begin{array}{c}\text { A Scalable and } \\
\text { Self-Configuring } \\
\text { Architecture for } \\
\text { Service Discovery in } \\
\text { the Internet of Things } \\
\text { [36] }\end{array}$ & $\begin{array}{c}\text { Discovery service for } \\
\text { large scale IOT } \\
\text { networks }\end{array}$ & $\begin{array}{c}\text { Based on P2P } \\
\text { technology and } \\
\text { Zeroconf methodology. } \\
\text { Make use of special } \\
\text { component called IoT } \\
\text { gateway to store } \\
\text { information on the } \\
\text { resources provided by } \\
\text { smart devices }\end{array}$ & Context awareness & Distributed \\
\hline 2014 & $\begin{array}{c}\text { A Data Sharing } \\
\text { Strategy and a DSL for } \\
\text { Service Discovery, } \\
\text { Selection and } \\
\text { Consumption for the } \\
\text { IoT [38] }\end{array}$ & $\begin{array}{c}\text { Discovery service } \\
\text { enforcing security in } \\
\text { terms of access } \\
\text { control }\end{array}$ & $\begin{array}{c}\text { Based on } \\
\text { query-response model }\end{array}$ & $\begin{array}{l}\text { Access Control is } \\
\text { given importance } \\
\text { instead of service } \\
\text { discovery }\end{array}$ & Distributed \\
\hline 2013 & $\begin{array}{c}\text { Adaptive and } \\
\text { Context-aware Service } \\
\text { Discovery for the } \\
\text { Internet of Things. [39] }\end{array}$ & $\begin{array}{l}\text { To overcome the } \\
\text { interoperability } \\
\text { problems in } \\
\text { constrained devices }\end{array}$ & $\begin{array}{l}\text { Registry based service } \\
\text { discovery solution with } \\
\text { context information } \\
\text { thereby it uses the } \\
\text { context information to } \\
\text { provide optimal service } \\
\text { selection } \\
\end{array}$ & $\begin{array}{l}\text { Importance is } \\
\text { towards IoT } \\
\text { deployments in } \\
\text { terms of device } \\
\text { processing power } \\
\text { and network } \\
\text { bandwidth. } \\
\end{array}$ & $\begin{array}{l}\text { Centralized } \\
\text { solution }\end{array}$ \\
\hline 2013 & $\begin{array}{c}\text { Constrained } \\
\text { Application Protocol } \\
\text { (CoAP)- RFC } 7252 \\
{[40]}\end{array}$ & $\begin{array}{c}\text { To avoid human } \\
\text { intervention in M2M } \\
\text { applications }\end{array}$ & $\begin{array}{l}\text { Discovery is through } \\
\text { exposing a } \\
\text { programmable interface }\end{array}$ & $\begin{array}{l}\text { The protocol lacks } \\
\text { with information } \\
\text { regarding how the } \\
\text { newly joining node } \\
\text { announce itself to } \\
\text { the network. }\end{array}$ & Distributed \\
\hline 2012 & $\begin{array}{l}\text { Convergence of utility } \\
\text { computing with the } \\
\text { internet-of-things [35] }\end{array}$ & $\begin{array}{c}\text { To deliver IoT } \\
\text { services on demand }\end{array}$ & $\begin{array}{l}\text { Uses ontology and } \\
\text { SPARQL languages for } \\
\text { performing discovery }\end{array}$ & $\begin{array}{l}\text { No focus on a } \\
\text { full-fledged } \\
\text { cloud platform } \\
\text { capable to host } \\
\text { third-party IoT } \\
\text { applications } \\
\text { and services } \\
\end{array}$ & "Distributed \\
\hline 2012 & $\begin{array}{c}\text { A DHT-based } \\
\text { discovery service } \\
\text { for the Internet of } \\
\text { Things [22] } \\
\end{array}$ & $\begin{array}{c}\text { To search the } \\
\text { resource using } \\
\text { multi-attribute and } \\
\text { range queries. } \\
\end{array}$ & $\begin{array}{c}\text { Uses DHT indexing } \\
\text { scheme. }\end{array}$ & $\begin{array}{c}\text { P2P overlay } \\
\text { creation is not } \\
\text { given importance. }\end{array}$ & Distributed \\
\hline 2012 & $\begin{array}{c}\text { Service discovery for } \\
\text { internet of things: a } \\
\text { context-awareness } \\
\text { perspective [33] } \\
\end{array}$ & $\begin{array}{c}\text { Environment aware } \\
\text { services for enabling } \\
\text { smart services to } \\
\text { things. } \\
\end{array}$ & $\begin{array}{l}\text { Ontology based } \\
\text { approach. }\end{array}$ & Limited scope & Distributed \\
\hline 2010 & $\begin{array}{l}\text { PDUS: P2P-based } \\
\text { distributed UDDI }\end{array}$ & $\begin{array}{l}\text { To overcome the } \\
\text { problems faced by }\end{array}$ & $\begin{array}{l}\text { Uses Chord DHT to } \\
\text { organize distributed }\end{array}$ & $\begin{array}{c}\text { Context awareness } \\
\text { support. }\end{array}$ & Distributed \\
\hline
\end{tabular}




\begin{tabular}{|c|c|c|c|c|c|}
\hline & $\begin{array}{c}\text { service discovery } \\
\text { approach [29] }\end{array}$ & $\begin{array}{c}\text { UDDI centralized } \\
\text { approach }\end{array}$ & 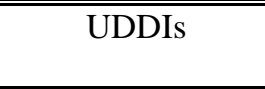 & & \\
\hline 2005 & $\begin{array}{c}\text { Distributed service } \\
\text { discovery with } \\
\text { guarantees in } \\
\text { peer-to-peer networks } \\
\text { using distributed hash } \\
\text { tables [30] }\end{array}$ & $\begin{array}{c}\text { To find the service } \\
\text { description matching } \\
\text { a given query without } \\
\text { flooding the entire } \\
\text { network }\end{array}$ & $\begin{array}{l}\text { Uses Chord DHT } \\
\text { indexing scheme }\end{array}$ & $\begin{array}{l}\text { Requirements for } \\
\text { IoT is not } \\
\text { addresses. }\end{array}$ & "Distributed. \\
\hline 1999 & $\begin{array}{c}\text { Service Location } \\
\text { Protocol, Version } 2 \\
{[34]}\end{array}$ & $\begin{array}{l}\text { To find services in } \\
\text { LANs without prior } \\
\text { configuration }\end{array}$ & $\begin{array}{l}\text { Uses Substring } \\
\text { matching feature }\end{array}$ & Prone to Failure & Centralized \\
\hline
\end{tabular}

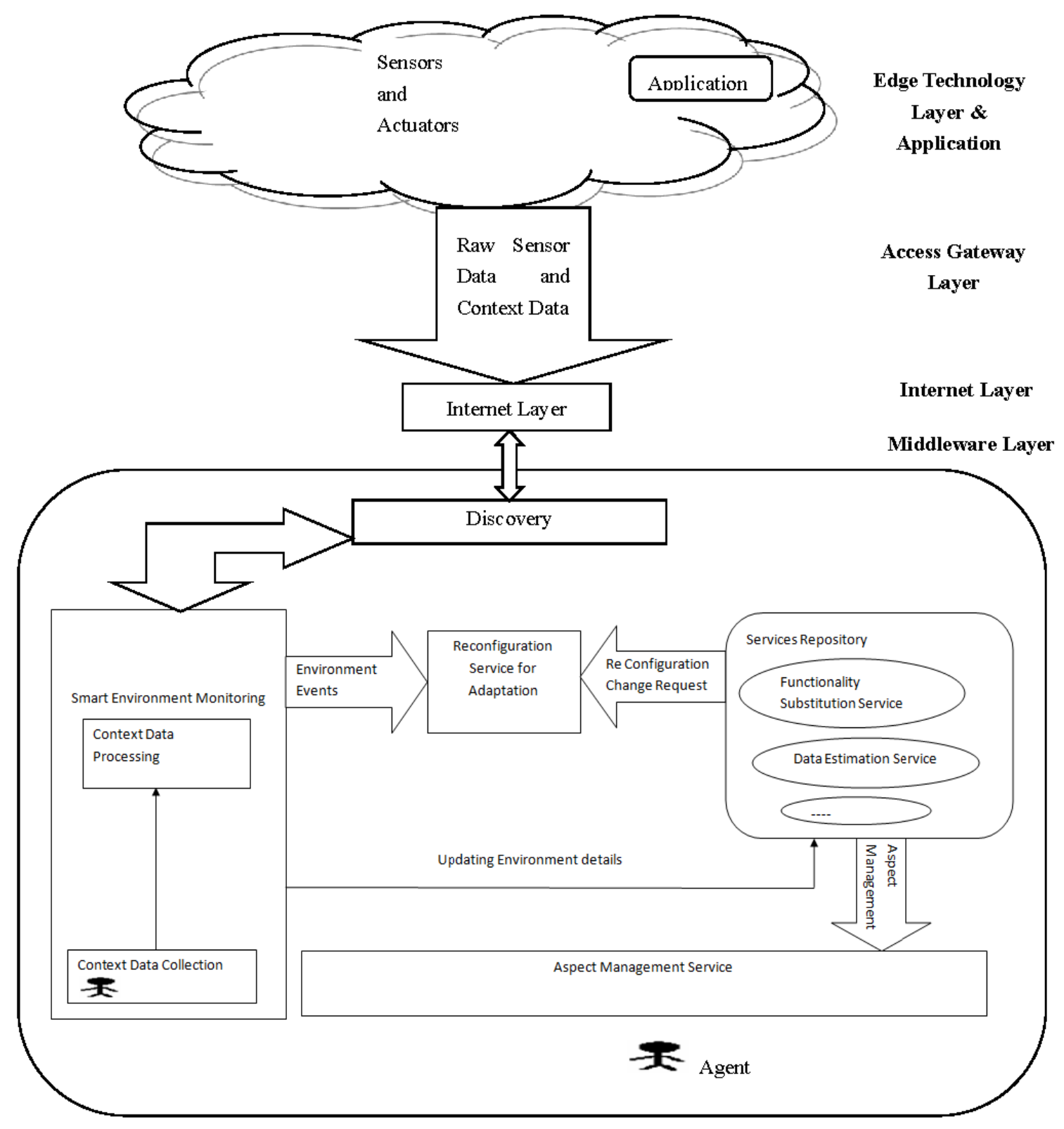

Figure 1 Middleware Framework for IoT 


\section{Discussion and Conclusion}

This work explored the literature review of service discovery algorithms in the context of Internet of Things paradigm. In particular we clarified service discovery fundamentals, features and techniques. The discussion reveals various protocols in the literature, features, usage, merits and pitfalls. The understanding of the various protocols was revealed in the tabular form. The final indication of suggested framework incurporating some of the issues with service discovery design and implementation was presented. Through the proposed middleware frame work, three main contribution are disseminated namely

- The application and infrastructure services are reconfigured based on the context data.

- The modelling of services addressing unknown topology and data availability issues as aspects are enabled and disabled according to the need.

- The middleware architecture is extensible thereby supporting addition and removal of services whenever necessary.

The middleware framework was tested for the class of small scale application that include temperature monitoring and motor vehicle monitoring system. The evaluation was done in the context of deploying appropriate functional substitution service while the parameters relevant to calculation are not available. In the motor vehicle tracking system the service was tested for locating the car at a particular time $\mathrm{t}_{1}$ such that $\mathrm{t}_{0}<$ $t_{1}<t_{2}$ where the location at time $t_{0}$ and $t_{2}$ are available. Evaluation of reconfiguration service was done in terms of evaluating Aspect Management Service using Spring AOP. The performance of reconfiguration approach was assessed not only by updating the parameters required for the service and also by adding and removing the service components. The assessment of application and middleware reconfiguration performance based on the context data provided by the mulitagent is the ongoing work and it requires the consideration of other non-functional requirements such as mobility and fault tolerance as these parameters are important for resource constrained devices. We understood that the analysis of the characteristics of examined approaches, its issues and challenges might help the future researchers to develop innovative approaches in this field.

\section{References}

[1] G. Tselentis, A. Galis, A. Gavras, S. Krco,V. Lotz, E. Simperl, B. Stiller, T. Zahariadis, "Towards the Future Internet-emerging trends from european research", IOS Press, 2010.

[2] V. Issarny, N. Georgantas, S. Hachem, A. Zarras, P. Vassiliadis, M. Autili, M. Gerosa, A. Ben Hamida, "Service-Oriented Middleware for the Future Internet: State of the Art and Research Directions", Journal of Internet Services and Applications, Vol.2, pp.23-45, 2011.

[3] CASAGRAS: RFID and the inclusive model for the internet of things. http://www.rfidglobal.eu, 2009

[4] P. Daras, D. Williams, C. Guerrero, I. Kegel, I. Laso,J. Bouwen, J.-D. Meunier, N. Niebert, and T. Zahariadis, "Why do we need a Content-Centric Future Internet? Proposals Towards ContentCentric Internet Architectures", European Commission, Networked Media Unit, Information Society and Media, May 2009

[5] D. Papadimitriou, "Future Internet-The Cross-ETP Vision Document", European Technology Platform, Alcatel Lucent, 2009.

[6] D. Guinard, V. Trifa, and E. Wilde, "A resource oriented architecture for the web of things", in Proceedings of the 2nd International Internet of Things Conference (IoT '10), pp. 9-129, December 2010 .

[7] Eleonora Borgia, "The Internet of Things vision: Key features, applications and open issues", Computer Communications, Vol.54, pp.1-31, 2014.

[8] IoT Definition http://www.rfidglobal.eu/userfiles/documents/CASA GRAS26022009.pdf

[9] Oleksiy Mazhelis, Martin Waldburger, Guilherme Sperb Machado, Burkhard Stiller, Pasi Tyrväinen, "Retrieving Monitoring and Accounting Information from Constrained Devices in Internet-ofThings Applications", in Emerging Management Mechanisms for the Future Internet, Lecture Notes in Computer Science, Vol.7943, pp.136-147, 2013.

[10]T.A. Butt, "Provision of adaptive and context-aware service discovery for the Internet of Things", Doctoral dissertation, Loughborough University, 2014.

[11]Badis Djamaa et al. "Service Discovery in 6LoWPANs: Classification \& Challenges", In Proceedings of 8th IEEE International Symposium on 
Service Oriented System Engineering, 2014

[12]Mehdi Khouja, Carlos Juiz, Ramon Puigjaner, and Farouk Kamoun, "Models for Service and Resource Discovery in Pervasive Computing", Pervasive Computing and Networking, pp.27-36, John Wiley \&Sons, 2011.

[13]S. Kaflle, K. Loesing, and G. Wirtz, "Distributed service discovery with guarantees in peer-to-peer networks using distributed hash tables", in Proceedings of International Conference on Parallel and Distributed Processing Techniques and Applications (PDPTA), pp.578-584, 2005.

[14]A. Passarella, "A survey on content-centric technologies for the current internet: $\mathrm{CDN}$ and P2P solutions", Computer Communications, Vol.35, pp.1-32, 2012.

[15]S. Ramabhadran, S. Ratnasamy, J.M. Hellerstein, S. Shenker, "Brief announcement: Prefixhash tree", in Proceedings of the 23th ACM Symposium on Principles of Distributed Computing, 2004.

[16]A.R. Bharambe, M. Agrawal, S. Seshan, "Mercury: supporting scalable multi-attribute range queries", in Proceedings of ACM SIGCOMM, pp.353-366, 2004 .

[17]M. Cai, M. Frank, J. Chen, P. Szekely, "MAAN: a multi-attribute addressable network for grid information services", in Proceedings of the 4th International Workshop on Grid Computing, GRID, 2003.

[18]C. Schmidt, M. Parashar, "Squid: enabling search in DHT-based systems", Journal of Parallel and Distributing Computing, Vol. 68, pp.962-975, 2008.

[19]F.Zhu, M.Mutka, L.M.Ni, (2005) "Service Discovery in Pervasive Computing Environments", IEEE Pervasive Computing, Vol.4, pp. 81-90, 2005.

[20]E.J. Project, Cloud of Things for empowering the citizen clout in smart cities, 2013-2016, http://cloutproject.eu/.

[21]Jara, J.Antonio, etal. "Mobile digcovery: discovering and interacting with the world through the Internet of things", Personal and ubiquitous computing, Springer, Vol. 18, pp. 323-338, 2014.

[22]F. Paganelli and D. Parlanti, "A DHT-based discovery service for the Internet of Things", Journal of Computer Networks and Communications, 2012 ,

[23]UPnP Forums, http://www.upnp.org/, 1999.
[24]S. Cheshire and M. Krochmal, "Multicast DNS (Internet Draft)", Apple Computer, Inc. http://files. multicastdns.org/draft-cheshire-dnsext-multicastdns .txt, 2004.

[25]Sun Microsystems,"JINI Architecture Specification", 1999.

[26]A. Rahman, E. Dijk, "Group Communication for CoAP. Internet Engineering Task Force, InternetDraftDraft-Ietf-Core-Groupcomm-24", http://tools. ietf.org/id/draft-ietf-core-groupcomm-24.txt, 2014.

[27]Salutation Consortium, "Salutation Architecture Specification",http://web.archive.org/web/2003062 3193812/www.salutation.org/, 1999. (The Salutation Consortium was disbanded on 30 June 2005).

[28]"Specification of the Bluetooth System", http:/ /www. bluetooth.com, December 1999.

[29]N. Yulin, S. Huayou, L. Weiping, and C. Zhong, "PDUS: P2P-based distributed UDDI service discovery approach", in Proceedings of International Conference on Services Science (ICSS), pp. 3-8, 2010.

[30]S. Kaflle, K. Loesing, and G. Wirtz, "Distributed service discovery with guarantees in peer-to-peer networks using distributed hash tables", in Proceedings of International Conference on Parallel and Distributed Processing Techniques and Applications (PDPTA), pp.578-584, 2005.

[31]Peter Wright, Yih Leong Sun, Terence Harmer, Anthony Keenan, Alan Stewart and Ronald Perrott, "A constraints-based resource discovery model for multi-provider cloud environments", Journal of Cloud Computing: Advances, Systems and Applications, Springer, pp-1-14, 2012.

[32]Ebbits Project, http://www.ebbits-project.eu

[33]Wei, Qiang, and ZhiJin. "Service discovery for internet of things: a context-awareness perspective", in Proceedings of the Fourth Asia-Pacific Symposium on Internet ware, ACM, 2012.

[34]E. Guttman, C. Perkins, J. Veizades, and M. Day, "Service Location Protocol, Version 2", IETF RFC 2608, June 1999.

[35]Soldatos, John, Martin Serrano, and Manfred Hauswirth. "Convergence of utility computing with the internet-of-things." in Proceedings of Sixth IEEE International Conference on Innovative Mobile and Internet Services in Ubiquitous Computing (IMIS), 2012.

[36]Cirani, Simone, Luca Davoli, Gianluigi Ferrari, 
RémyLéone, Paolo Medagliani, Marco Picone, and Luca Veltri. "A Scalable and Self-Configuring Architecture for Service Discovery in the Internet of Things.", IEEE Internet of Things Journal, Vol.1 , 2014.

[37]Apple Inc,"Bonjour Technology White Paper", Available: http://images.apple.com/macosx/pdf/Mac OSX_Bonjour_TB.pdfT, 2007.

[38]Adda, Mehdi, and RabebSaad. "A Data Sharing Strategy and a DSL for Service Discovery, Selection and Consumption for the IoT." Procedia Computer Science 37, pp. 92-100, 2014.

[39]Butt, Talal Ashraf, Iain Phillips, Lin Guan, and George Oikonomou. "Adaptive and Context-aware Service Discovery for the Internet of Things", Internet of Things, Smart Spaces, and Next Generation Networking, Springer Berlin Heidelberg, pp. 36-47, 2013.

[40]Z.Shelby, K.Hartke, and C.Bormann. (Jun.2013). Constrained Application Protocol (CoAP). RFC 7252 (Proposed Standard), Internet Engineering Task Force, http://tools.ietf.org/html/rfc7252

[41]OpenIoT Project, http://openiot.eu

\section{[42]UDDI: http://uddi.microsoft.com}

[43]C.N.Ververidis,G.C.Polyzos,"Service disco- very for mobile ad hoc networks: a survey of issues and techniques", IEEE Communications Surveys, Vol. 10, 2008.

[44]D. Chakraborty, A. Joshi, Y. Yesha, Y, T. Finin, "Toward distributed service discovery in pervasive computing environments", IEEE Transactions on Mobile Computing, Vol. 5, 2006.

[45]Z. Gao, L. Wang, M. Yang, X. Yang, "CNPGSDP: an efficient group-based service discovery protocol for MANETs", Computer Networks, Vol. 50, 2006.

[46]Z. Gao, L. Wang, X. Yang, D. Wen, "PCPGSD: an enhanced GSD service discovery protocol for MANETs", Computer Communications, Vol.29, 2006.

[47]Z. Gao, X. Yang,T. Ma, S. Cai, "RICFFP: An e?cient service discovery protocol for MANETs", Embedded and Ubiquitous Computing, LNCS, Vol. 3207, 2004.

[48]P.Grace, G.Blair, S.Samuel, "ReMMoC: A rerective middle ware to support mobile client interoperability", On The Move to Meaningful Internet Systems, LNCS, Vol. 2888, 2003.
[49]Y.D. Bromberg,V.Issarny, "INDISS: Interoperable discovery system for networked services", in Proceedings of ACM/IFIP/USENIX International Conference on Middleware, 2005.

[50]P.G.Raverdy, V.Issarny, R.Chibout, A. de La Chapelle, "A multi-protocol approach to service discovery and access in pervasive environments", in Proceedings of 3rd Annual International Conference on Networking Services, Mobile and Ubiquitous Systems, 2006.

[51]D.Cotroneo, A.Graziano, S.Russo, "Security requirements in service oriented architectures for ubiquitous computing", in Proceedings of 2 nd Workshop on Middleware for Pervasive and Ad-Hoc Computing (MPAC), 2004.

[52]M. Nidd, "Service discovery in DEAP space", IEEE Personal Communications, Vol.8, 2001.

[53]S.De et al. "Concepts and Solutions for Entitybased Discovery of IoT Resources and Managing their Dynamic Associations", IoT-A Deliverable D4.3, 2012.

[54]S. De, B. Christophe, K. Moessner, "Semantic enablers for dynamic digital physical object associations in a federated node architecture for the internet of things", Ad Hoc Networks, Vol.18, pp. 102-120, 2014.

[55]J.Boss-AOP: (http://www.jboss.org/jbossaop) Last visited: February, 2011.

[56]M. A. Chaqfeh, N. Mohamed, P. O. Box, and A. Ain, "Challenges in Middleware Solutions for the Internet of Things", in Proceedings of IEEE International conference on Collaboration Technologies and Systems (CTS), pp. 21-26, 2012.

[57]G.Mulligan, "An Interaction Independence Middleware Framework", Virginia Polytechnic Institute and State University, 2009.

[58]T.Teixeira, S. Hachem, and N. Georgantas, "Service Oriented Middleware for the Internet of Things: A Perspective", in Towards a Service-Based Internet, Vol.257178, No. 257178, J. Abramowicz, Witold and Llorente, IgnacioM. And Surridge, Mike and Zisman, Andrea and Vayssière, Ed. Springer Berlin Heidelberg, pp. 220-229, 2011. 